\title{
Brain-derived and glial cell line-derived neurotrophic factor fusion protein immobilization to laminin
}

\author{
BAOXIN WANG $^{1 *}$, JUNJIE YUAN $^{2 *}$, JIAFENG XU $^{3}$, XINWEI CHEN $^{1}$, XINJIANG YING $^{1}$ and PIN DONG ${ }^{1}$ \\ ${ }^{1}$ Department of Otolaryngology, Head and Neck Surgery, Shanghai Jiao Tong University Affiliated to Shanghai First People's \\ Hospital, Shanghai 201620; ${ }^{2}$ Department of Orthopedics, Shanghai Fengxian District Central Hospital, \\ Shanghai Jiao Tong University Affiliated to Sixth People's Hospital South Campus, Shanghai 200011; \\ ${ }^{3}$ School of Economics and Finance, Shanghai International Studies University, Shanghai 200083, P.R. China
}

Received July 8, 2015; Accepted September 1, 2016

DOI: $10.3892 / \mathrm{etm} .2016 .3925$

\begin{abstract}
Damage to the recurrent laryngeal nerve often causes hoarseness, dyspnea, dysphagia, and sometimes asphyxia due to vocal cord paralysis which result in a reduction of quality of life. Brain-derived neurotrophic factor (BDNF) and glial cell line-derived neurotrophic factor (GDNF) play critical roles in peripheral nerve regeneration. However, methods for efficiently delivering these molecules are lacking, which limits their use in clinical applications. The present study reports an effective strategy for targeting BDNF and GDNF to laminin by fusing the N-terminal domains of these molecules with agrin (NtA). More specifically, laminin-binding efficacy was assessed and sustained release assays of the delivery of BDNF or GDNF fused with NtA (LBD-BDNF or LBD-GDNF) to laminin were conducted in vitro. In addition, the bioactivity of LBD-BDNF and LBD-GDNF on laminin in vitro was investigated. LBD-BDNF and LBD-GDNF were each able to specifically bind to laminin and maintain their activity in vitro. Moreover, neurotrophic factors with NtA retained higher concentrations and bioactivity levels compared with those without NtA. The ratio of LBD-BDNF and LBD-GDNF that produced optimal effects was 4:6. BDNF and GDNF fused with NtA were effective in specifically binding to laminin. As laminin is a major component of the extracellular matrix, LBD-BDNF and LBD-GDNF may prove useful in the repair of peripheral nerve injuries.
\end{abstract}

Correspondence to: Dr Pin Dong, Department of Otolaryngology, Head and Neck Surgery, Shanghai Jiao Tong University Affiliated to Shanghai First People's Hospital, 100 Haining Road, Shanghai 201620, P.R. China

E-mail: dongpin64@aliyun.com

*Contributed equally

Key words: brain-derived neurotrophic factor, glial cell line-derived neurotrophic factor, immobilization, laminin, PC12 cell

\section{Introduction}

The recurrent laryngeal nerve (RLN) can be damaged during surgery of the head and neck (1). One of the most significant symptoms of damage to this nerve is incomplete glottic closure, which can profoundly decrease quality of life (2). Medialization procedures are commonly carried out to treat unilateral vocal fold palsy, solely fulfilling vocal fold medialization by static changes in the laryngeal framework (3). Neurological impairment can be compensated for by the regeneration of peripheral nerves. However, experimental and clinical evidence has shown that, particularly after severe injuries, regenerative ability is usually limited with unsatisfactory results (1,4-6). Thus, in order to improve recovery following nerve injury, one therapeutic option is to use natural stimulatory reagents, such as neurotrophic factors.

Neurotrophic factors are able to enhance nerve regeneration, and therefore may be useful as a molecular therapy. Among all neurotrophic factors, brain-derived neurotrophic factor (BDNF) and glial cell line-derived neurotrophic factor (GDNF) play critical roles. BDNF promotes angiogenesis and neural regeneration, as well as modulates local inflammatory processes (7). GDNF has been shown to boost nerve regeneration after injury and exerts survival-promoting effects on motor neurons in vivo and in vitro (8-11). However, in practice, a large proportion of neurotrophic factors are diffused away from the site of injury; thus, it is difficult to retain effective concentrations of such factors due to their rapid diffusion in extracellular fluids. One solution to this problem is repeated injections of neurotrophic factors to the injured sites so that therapeutic concentrations can be obtained; however, this strategy poses unnecessary risks and costs (12). Thus, the discovery of a better approach for targeting neurotrophic factors to wound sites is imperative (13).

One method of overcoming the diffusion of neurotrophic factors is by taking advantage of their binding affinity to certain molecules. In doing so, these factors can be immobilized at the site of injury. The glycoprotein, laminin, appears to be an appropriate material for targeting damaged sites because it is a major component of the extracellular matrix (ECM) and is biocompatible (13). Laminin is mainly produced by Schwann cells and is widespread in the peripheral nervous 
system (PNS) $(14,15)$. Following injury to the PNS, laminin is upregulated and promotes axonal regeneration $(16,17)$. It has been reported that BDNF has an affinity for laminin, but this affinity is low; more than half of BDNF bound to laminin is quickly released within the first day of injury (13). Thus, the native affinity of BDNF for laminin appears to be insufficient for therapeutic purposes.

Previous studies have demonstrated that the N-terminal domain of agrin (NtA) has a high affinity to laminin (18). Making use of this so-called laminin-binding domain (LBD), a tripartite fusion protein, which included a six-histidine purification tag, LBD, and the sequence of native BDNF or GDNF was made in the present study. Thus, the two fusion proteins that resulted were named LBD fused BDNF (LBD-BDNF) and LBD fused GDNF (LBD-GDNF). A native BDNF and GDNF without NtA, designated NAT-BDNF and NAT-GDNF, respectively, were prepared as controls. The overall aim of this study was to utilize laminin as a binding target so that a delivery system could be constructed to maintain neurotrophic factor concentrations within certain regions of interest.

\section{Materials and methods}

Preparation of recombinant proteins. LBD-BDNF was prepared as previously described (13), and LBD-GDNF was constructed in an analogous manner. GDNF DNA, extracted from the rat cell line PC12 [purchased from the Cell Bank of the Chinese Academy of Sciences (Shanghai, China)], was amplified by polymerase chain reaction (PCR) with a KOD Plus polymerase kit (Toyobo Co., Ltd., Osaka, Japan). Primer sequences used were as follows: Forward, GGTAGC GGCAGCGGTAGCACATGCCCGGAGCGCGCGCTG; reverse, TACTCGAGTCAGATACATCCACACCTTTTAG. Reaction conditions for PCR were as follows: Initial denaturation, $95^{\circ} \mathrm{C}$ for $5 \mathrm{~min}$; 30 cycles of denaturation at $95^{\circ} \mathrm{C}$ for $30 \mathrm{sec}$, annealing at $60^{\circ} \mathrm{C}$ for $30 \mathrm{sec}$ and extension at $68^{\circ} \mathrm{C}$ for $30 \mathrm{sec}$; followed by a final extension at $68^{\circ} \mathrm{C}$ for $5 \mathrm{~min}$. and inserted into the vectors, pET-LBD and pET-28a (Novagen; EMD Millipore, San Diego, CA, USA). Escherichia coli BL21 (DE3) (Biovector Science Lab, Inc., Beijing, China) was then transformed with the vectors, and proteins were induced with $1 \mathrm{mM}$ isopropyl $\beta$-D-thiogalactopyranoside (IPTG) at $37^{\circ} \mathrm{C}$ for $5 \mathrm{~h}$. Proteins were accumulated in inclusion bodies, and purification of the solubilized proteins was performed under denaturing conditions by nickel chelate chromatography, using imidazole-containing buffer, with a HiTrap Chelating High Performance column and an AKTA fast protein liquid chromatography system (Amersham; GE Healthcare, Uppsala, Sweden). The purity of the recombinant proteins was analyzed by $15 \%$ (wt/vol) sodium dodecyl sulfate-polyacrylamide gel electrophoresis (SDS-PAGE), by which $20 \mu \mathrm{g}$ of protein was separated and stained with Coomassie brilliant blue solution (Beyotime Institute of Biotechnology, Haimen, China) for $1 \mathrm{~h}$, then washed in saline, or transferred to a PVDF membrane. This was followed by a block in 5\% milk solution, then western blotting with a mouse antibody against polyhistidine (cat. no. ab18184; 1:2,000 dilution; Abcam, Cambridge, UK). This was subsequently incubated with horseradish peroxidase-conjugated goat anti-mouse antibody (cat. no. ab97040; 1:8,000 dilution; Abcam) for $2 \mathrm{~h}$ at room temperature, following which bands were visualized with enhanced chemiluminescence reagent (Thermo Fisher Scientific, Inc., Waltham, MA, USA). Purified proteins were then dialyzed in a glutathione redox-refolding system by a chromatographic method (19) and stored at $-80^{\circ} \mathrm{C}$.

In vitro laminin-binding assay. In order to measure laminin binding capacity, a modified enzyme-linked immunosorbent assay (ELISA) technique was employed in which laminin (Sigma-Aldrich; Merck Millipore, Darmstadt, Germany) was added to 96-well plates (Costar). The plates were then incubated at $4^{\circ} \mathrm{C}$ for $24 \mathrm{~h}$, and ventilated to dry. After washing the plates with phosphate-buffered saline (PBS; $\mathrm{pH}$ 7.3) five times, the plates were blocked with $2.5 \%$ (wt/vol) bovine serum albumin (Wuhan Boster Biological Technology, Ltd.- Wuhan, China) containing $0.1 \%$ ( $\mathrm{vol} / \mathrm{vol}$ ) Tween 20 at $37^{\circ} \mathrm{C}$ for $2 \mathrm{~h}$. The four recombinant proteins with increasing concentrations $(0-4 \mu \mathrm{M})$ were added to the plates $(100 \mu \mathrm{l} /$ well $)$ and incubated at $37^{\circ} \mathrm{C}$ for $2 \mathrm{~h}$. The plates were then washed five times with PBS to remove redundant proteins. Proteins bound to laminin were tested using mouse antibody against polyhistidine (cat. no. H1029; 1:1,000 dilution; Sigma-Aldrich) for $1 \mathrm{~h}$ at $37^{\circ} \mathrm{C}$, followed by goat antibody to mouse IgG (alkaline phosphatase-conjugated; cat. no. P7998; 1:10,000; Sigma-Aldrich) for $1 \mathrm{~h}$ at $37^{\circ} \mathrm{C}$. Bound proteins were detected using the alkaline phosphatase (AP) reaction with para-nitrophenylphosphate (pNPP; Sigma-Aldrich) in AP buffer ( $\mathrm{pH}$ 9.6) for $15 \mathrm{~min}$ at $37^{\circ} \mathrm{C}$; this reaction was stopped using $3 \mathrm{M} \mathrm{NaOH}$. Optical density at $405 \mathrm{~nm}$ (OD405) was quantified using an ELISA reader (Molecular Devices, LLC, Sunnyvale, CA, USA).

In vitro laminin releasing assay. Laminin loaded with $1.5 \mu \mathrm{mol} / 1 \mathrm{NAT}-\mathrm{BDNF}$ or LBD-BDNF or $3 \mu \mathrm{mol} / 1 \mathrm{NAT}-\mathrm{GDNF}$ or LBD-GDNF was placed in a 48-well plate. Laminin suspension was induced by the addition of $500 \mu \mathrm{l}$ PBS and incubation on a rocking platform $\left(37^{\circ} \mathrm{C}, 80 \mathrm{rpm}\right)$. PBS was changed every $24 \mathrm{~h}$, and at days $0-7$, samples were collected and the four proteins retained on the laminin were analyzed using the ELISA assay.

In vitro bioactivity assay. The biological activity of NAT-BDNF, LBD-BDNF, NAT-GDNF and LBD-GDNF was measured by neurite outgrowth and survival of PC12 cells (20). A density of $3 \times 10^{3}$ cells/well was seeded in polylysine-treated 96-well plates (Costar) and cultured in serum-free RPMI-1640 medium (Gibco; Thermo Fisher Scientific, Inc.) at $37^{\circ} \mathrm{C}$ for $1 \mathrm{~h}$. Increasing concentrations of NAT-BDNF, LBD-BDNF, NAT-GDNF or LBD-GDNF (0-800 ng) were then added to the plates. After $24 \mathrm{~h}$ of culture, the percentage of cells with neurites and the neurite length/ cell diameter ratio of cells containing neurites were determined. The number of surviving cells was determined using a Cell Counting kit (CCK)- 8 assay (Dojindo, Kumamoto, Japan) after $48 \mathrm{~h}$ of culture. Cells cultured under identical conditions without recombinant proteins served as a control. The results were evaluated in duplicate by two independent researchers.

In vitro bioactivity assay on laminin. Recombinant proteins were separately added to a 96-well plate coated with laminin and incubated at $4^{\circ} \mathrm{C}$ for $12 \mathrm{~h}$. Plates were then washed three 
times to remove any unbound BDNF or GDNF. PC12 cells were seeded at a density of $3 \times 10^{3}$ cells/well under the same conditions as described in the in vitro bioactivity assay. After $24 \mathrm{~h}$ of incubation, the cells were observed using phase contrast microscopy (Olympus Corporation, Tokyo, Japan), and the percentage of cells with neurites and length neurite/diameter of cells containing neurites were calculated. The number of surviving cells was examined using a CCK-8 assay after $48 \mathrm{~h}$ of culture. For controls, cells were cultured under the same conditions but without recombinant proteins.

Bioactivity of different proportions of $L B D-B D N F$ and $L B D-G D N F$ on laminin in vitro. Different proportions of LBD-BDNF and LBD-GDNF (1:4, 2:3, 3:2 and 4:1) were added to a 96-well plate coated with laminin, with the total protein added equaling 1,000 ng. The remainder of the steps were carried out as previously described in the bioactivity assay on laminin. Blank controls were manufactured using the abovementioned conditions, with the exception that one of the fusion factors was omitted.

Cytoskeletal staining. Different proportions of LBD-BDNF and LBD-GDNF (1:4, 2:3, 3:2 and 4:1) were added to a confocal dish coated with laminin. PC12 cells were seeded at a density of $3 \times 10^{3}$ under the same conditions as described in the bioactivity assay on laminin. The cells were washed three times with PBS and fixed with $4 \%$ paraformaldehyde for $2 \mathrm{~h}$ at room temperature. Cells were then incubated with $0.5 \mu \mathrm{g} / \mathrm{ml}$ FITC-phalloidin (Sigma-Aldrich) for $20 \mathrm{~min}$, followed by three washes with $0.3 \%$ Triton X-100. In order to identify cell nuclei, $0.1 \mu \mathrm{g} / \mathrm{ml}$ 4',6-diamidino-2-phenylindole (DAPI; Sigma-Aldrich) staining was used. Finally, after three washes in PBS to remove unbound DAPI, cells were observed using confocal fluorescent microscopy (Olympus Corporation).

Statistical analysis. All data were summarized as the mean value \pm standard deviation. A student's t-test was performed to analyze paired samples and one-way analysis of variance was performed to analyze multiple comparison procedures using Statistical Package for the Social Sciences (SPSS) version 20.0 software (IBM SPSS, Armonk, NY, USA). P $<0.05$ was considered to indicate a statistically significant difference.

\section{Results}

Fusion protein structures. The functional modules of NAT-BDNF, NAT-GDNF, LBD-BDNF and LBD-GDNF are shown in Fig. 1. After being induced by IPTG, the recombinant proteins were expressed, and identified by western blot analysis (Fig. 2).

LBD-BDNF and LBD-GDNF bind to laminin and exhibit sustained released in vitro. The binding of NAT-BDNF, LBD-BDNF, NAT-GDNF and LBD-GDNF to laminin was measured in vitro through a modified ELISA technique. From the results, it was concluded that the OD405 of neurotrophic factors with NtA (the LBD) was significantly higher than that of neurotrophic factors without NtA at each indicated point. These findings demonstrate that the retention of neurotrophic factors with $\mathrm{NtA}$ on laminin was significantly greater than
A

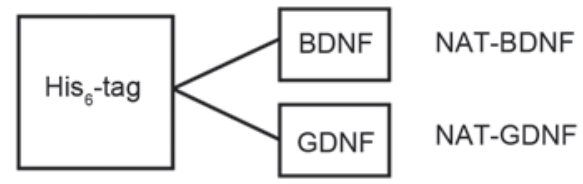

B

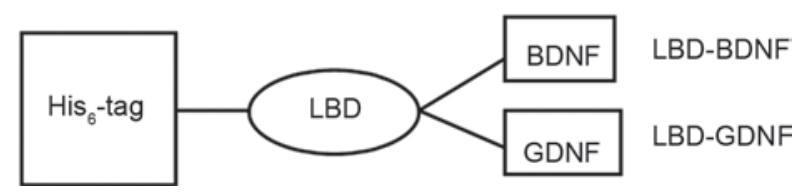

Figure 1. Schematic drawing of the engineered (A) NAT-BDNF, NAT-GDNF (B) LBD-BDNF and LBD-GDNF proteins. $\mathrm{His}_{6}$, six histidine (an affinity tag used to purify proteins); LBD, laminin-binding domain; BDNF, brain-derived neurotrophic factor (functional domain); GDNF, glial cell line-derived neurotrophic factor (functional domain); NAT, native.

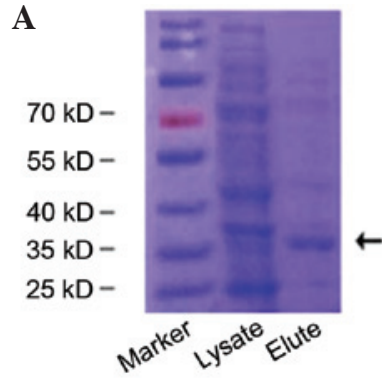

C

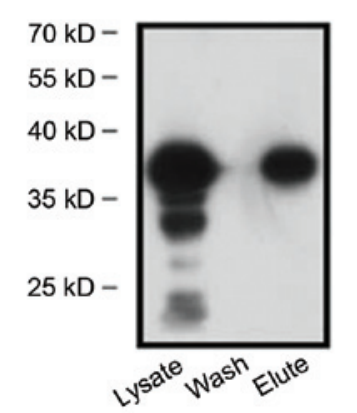

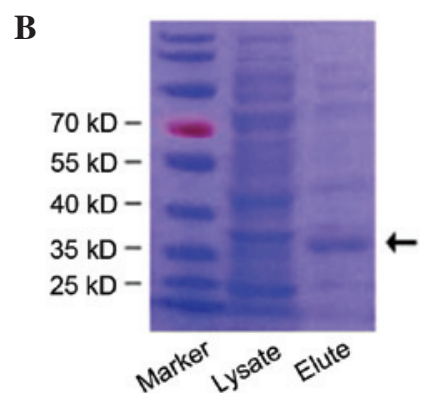

D

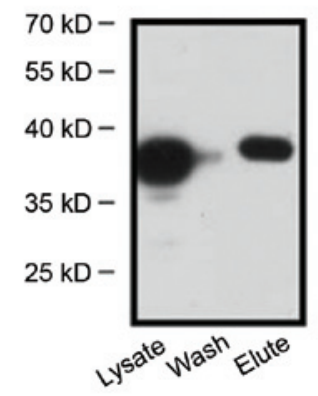

Figure 2. Sodium dodecyl sulfate polyacrylamide gel electrophoresis of purified proteins. Coomassie blue staining was used to detect the purification of (A) LBD-BDNF and (B) LBD-GDNF. Western blotting with an antibody to polyhistidine was then used to evaluate (C) LBD-BDNF and (D) LBD-GDNF. LBD, laminin-binding domain; BDNF, brain-derived neurotrophic factor; GDNF, glial cell line-derived neurotrophic factor.

that of neurotrophic factors without NtA $(n=6, P<0.05$; Fig. 3). Thus, LBD-BDNF and LBD-GDNF could specifically bind to laminin, and factors with NtA possessed stronger laminin-binding capacity.

In the in vitro release experiment, sustained release of neurotrophic factors was assessed for 7 days (Fig. 4). It was found that NAT-BDNF and NAT-GDNF were quickly released on day 1, whereas LBD-BDNF and LBD-GDNF were gradually released from day 1 to day 7 . During the 7 days, the quantities of LBD-BDNF and LBD-GDNF retained on laminin were significantly greater than those of NAT-BDNF and NAT-GDNF, respectively $(\mathrm{n}=6, \mathrm{P}<0.05)$. At day 7 , the percentage of NAT-BDNF and NAT-GDNF released from laminin was $\sim 87$ and $93 \%$, respectively, whereas the percentage 
A

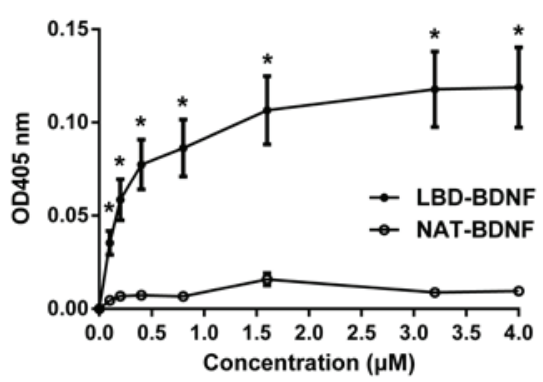

B

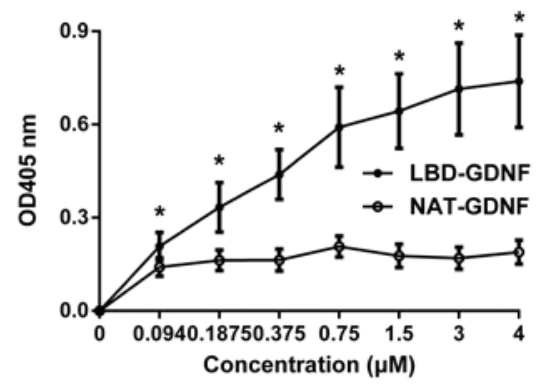

Figure 3. Laminin binding assay in vitro. Binding curves of (A) NAT-BDNF and LBD-BDNF and (B) NAT-GDNF and LBD-GDNF to laminin measured by enzyme-linked immunosorbent assay. $\mathrm{n}=6$. " $\mathrm{P}<0.05$ vs. NAT control. NAT, native; LBD, laminin-binding domain; BDNF, brain-derived neurotrophic factor; GDNF, glial cell line-derived neurotrophic factor; OD, optical density.

A

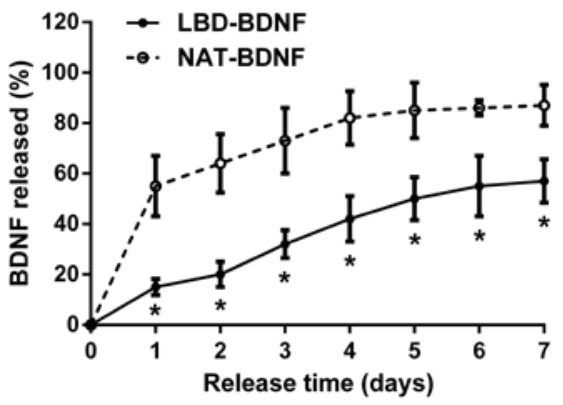

B

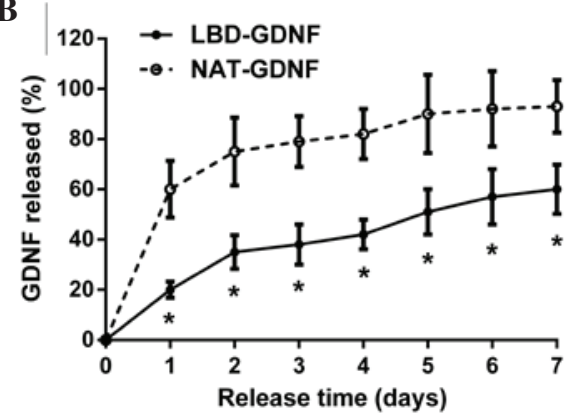

Figure 4. Sustained release assay from laminin in vitro. Release curves of (A) NAT-BDNF and LBD-BDN and (B) NAT-GDNF and LBD-GDNF from laminin in vitro. $\mathrm{n}=6 .{ }^{*} \mathrm{P}<0.05$ vs. NAT control. NAT, native; LBD, laminin-binding domain; BDNF, brain-derived neurotrophic factor; GDNF, glial cell line-derived neurotrophic factor.

A

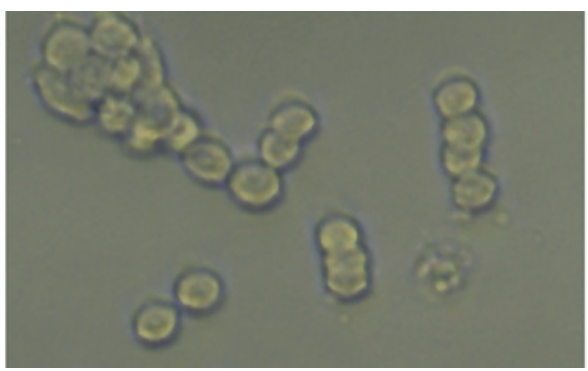

B

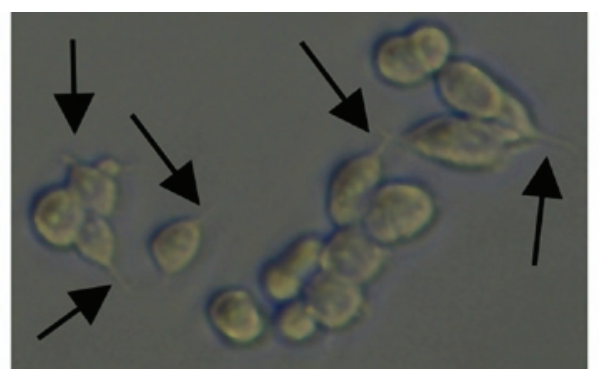

Figure 5. Characterization of PC12 cells was conducted by phase contrast microscopy for cells that were cultured under conditions (A) without recombinant proteins or (B) with LBD-BDNF. Black arrows indicate the neurites. Magnification, x200. LBD, laminin-binding domain; BDNF, brain-derived neurotrophic factor.

of LBD-BDNF and LBD-GDNF released from laminin was $\sim 57$ and $60 \%$, respectively. These results suggest that neurotrophic factors with a LBD can be retained on laminin for a longer time in vitro than those without.

LBD-BDNF and LBD-GDNF maintain higher bioactivity on laminin in vitro. $\mathrm{PC} 12$ cells were used to test the bioactivity of NAT-BDNF, LBD-BDNF, NAT-GDNF and LBD-GDNF. Fig. 5A shows PC12 cells cultured under general conditions, while Fig. 5B shows cells cultured with LBD-BDNF. PC12 cells were observed after $48 \mathrm{~h}$ incubation, and it was determined that the four factors significantly promoted neurite outgrowth and neuronal survival (Fig. 6), while there was no significant difference between them at each concentration (Fig. 6). This implies that the fusion of neurotrophic factors with a LBD moiety did not impair the inherent activity of BDNF or GDNF.

The bioactivities of BDNF and GDNF on laminin were then measured in vitro (Fig. 7). When neurotrophic factors were incubated in laminin-coated wells and PC12 cells were plated, a significant difference in growth promotion (the percentage of cells with neurites) compared with controls was observed (Fig. 7A and B). Greater numbers of living cells and longer neurites were also found for the neurotrophic factors with the LBD NtA ( $n=6, \mathrm{P}<0.05$; Fig. 7C-F). LBD-BDNF and LBD-GDNF maintained higher bioactivities, as well as greater concentrations on laminin than did the native controls. These results suggest that LBD-BDNF and LBD-GDNF can target laminin, and that these fusion proteins could be useful in nerve injury repair. Furthermore, when the ratio of LBD-BDNF and 
A

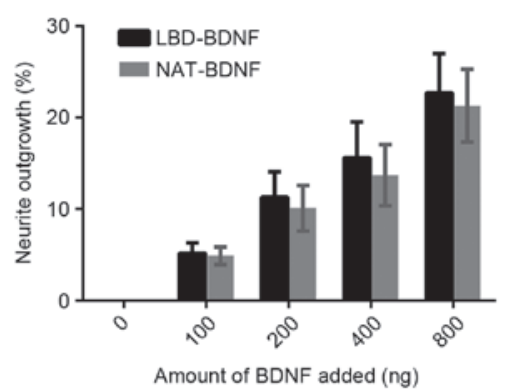

C

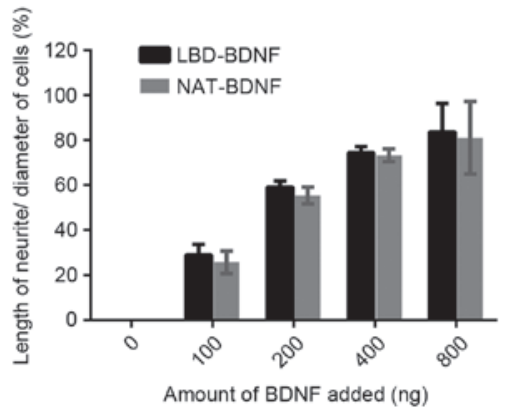

$\mathbf{E}$

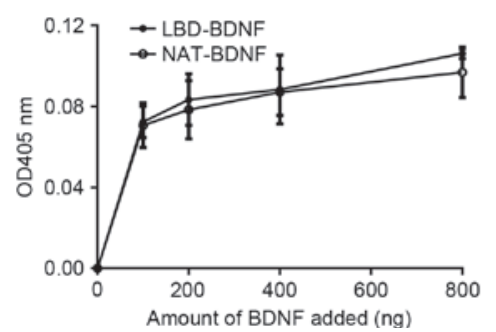

B

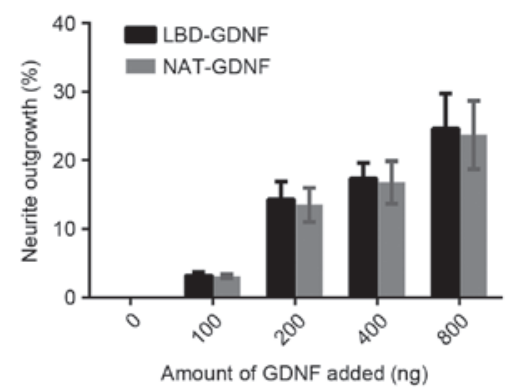

D

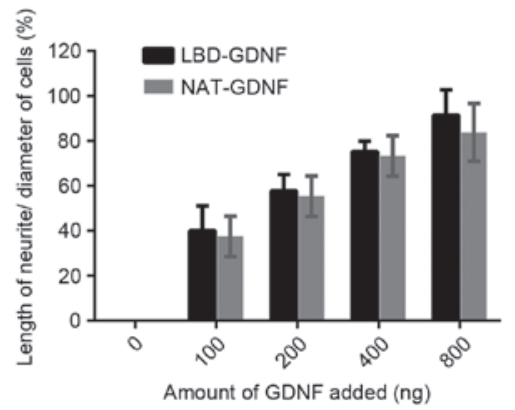

F

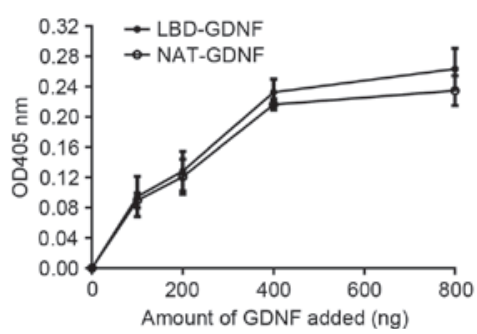

Figure 6. Bioactivity comparison of recombinant proteins in vitro. Effect of (A) LBD-BDNF, NAT-BDNF, (B) LBD-GDNF and NAT-GDNF on neurite outgrowth from PC12 cells. Effect of (C) LBD-BDNF, NAT-BDNF, (D) LBD-GDNF and NAT-GDNF on the ratio of neurite length/cell diameter in PC12 cells. Effect of (E) LBD-BDNF, NAT-BDNF, (F) LBD-GDNF and NAT-GDNF on PC12 cell survival as determined by Cell Counting kit-8 assay. Data are presented as mean \pm standard deviation $(\mathrm{n}=6)$. NAT, native; LBD, laminin-binding domain; BDNF, brain-derived neurotrophic factor; GDNF, glial cell line-derived neurotrophic factor; OD, optical density.

LBD-GDNF was $4: 6$, the ability to promote neurite growth was superior to that of the other ratios examined (Fig. 8).

Cytoskeletal staining. As shown in Fig. 9, the cytoskeleton, nucleus and neurites of the PC12 cells are clearly presented. Although the entire cell skeleton cannot be visualized, the featured images were chosen for their clear presentations of the neurites. After combining LBD-BDNF and LBD-GDNF, a greater effect was observed than when they were applied separately. Through these results, it may be concluded that when the ratio of mixing of LBD-BDNF and LBD-GDNF was 4:6, their ability to promote growth was superior to that at other ratios, demonstrating a synergetic effect (Fig. 8).

\section{Discussion}

Peripheral nerve injury often causes a loss of function, and the current gold standard for repairing such damage is autologous nerve grafting (21). However, the clinical application of autologous nerve grafting is hampered due to limited donor sites, extra incisions required, and a possible loss of function at the donor site (21). One potential therapeutic strategy that could address these issues is the application of natural stimulatory factors, such as neurotrophic factors, to the site of injury.

Neurotrophic factors are produced by organisms to promote neural cell survival, growth and differentiation. Neurotrophic factors are not only able to reduce nerve degeneration and prevent the progression of disease, but are also able to stimulate axonal growth and promote functional regeneration (22). Researchers have studied several fusion neurotrophic factors, including native human nerve growth factor (NGF)- $\beta$ fused with a collagen-binding domain (CBD) (23-25), fibronectin fused with CBD (26), BDNF fused with CBD (7,27-32), NGF- $\beta$ fused with LBD (20), ciliary neurotrophic factor fused with LBD (33), and BDNF fused with LBD (13); however, to the best of our knowledge, to date there have been no studies investigating an LBD-GDNF fusion protein.

BDNF and GDNF play important roles in the repair of nerve injury. However, it is difficult to retain them at injury sites because they easily diffuse. In order to maintain active concentrations, previous studies have employed multiple injections, adenoviral vectors overexpressing BDNF or GDNF, or fusion proteins such as CBD-BDNF and LBD-BDNF $(3,7,13,34,35)$. However, the risk of surgery, immunological rejection, and 
A

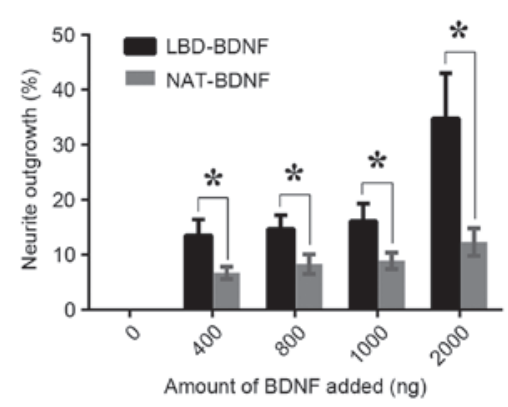

C

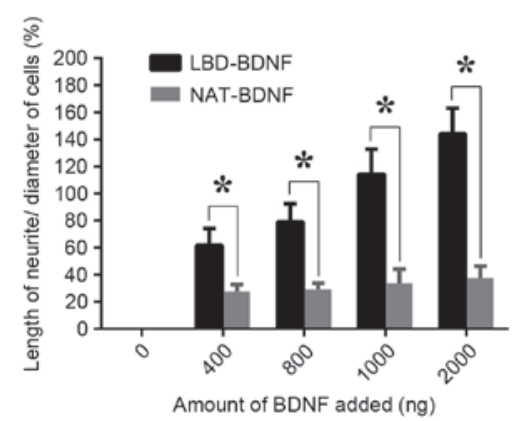

E

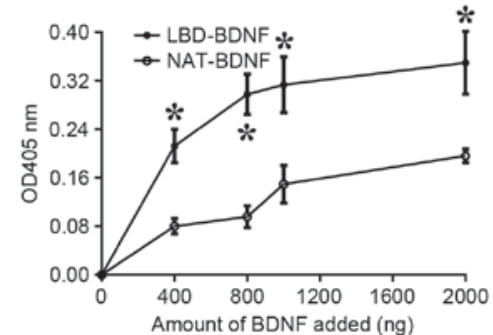

B

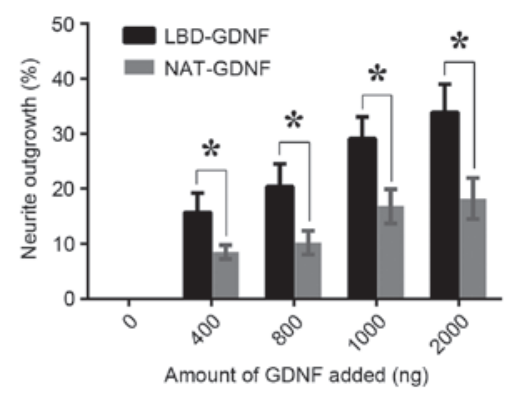

D

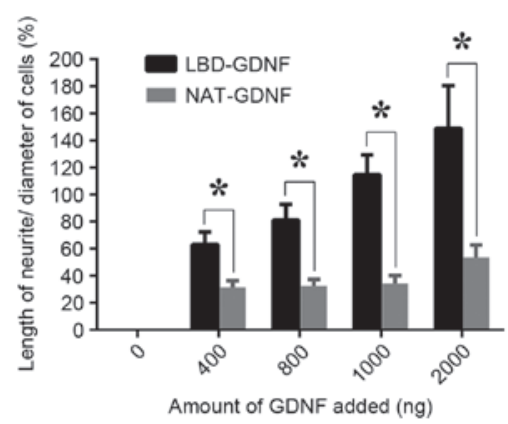

F

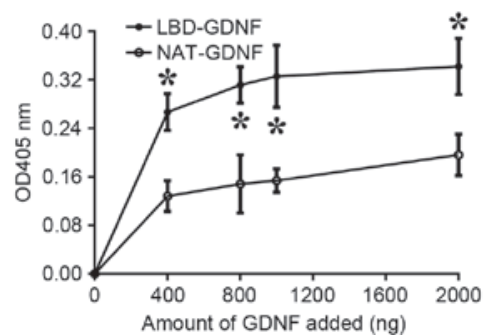

Figure 7. Bioactivity comparison of recombinant proteins on laminin in vitro. Effect of (A) LBD-BDNF, NAT-BDNF, (B) LBD-GDNF and NAT-GDNF on neurite outgrowth from PC12 cells. Effect of (C) LBD-BDNF, NAT-BDNF, (D) LBD-GDNF and NAT-GDNF on the ratio of neurite length/cell diameter in PC12 cells. Effect of (E) LBD-BDNF, NAT-BDNF, (F) LBD-GDNF and NAT-GDNF on PC12 cell survival as determined by Cell Counting kit-8 assay. Data are presented as mean \pm standard deviation $(\mathrm{n}=6)$. ${ }^{*} \mathrm{P}<0.05$ the NAT control. NAT, native; LBD, laminin-binding domain; BDNF, brain-derived neurotrophic factor; GDNF, glial cell line-derived neurotrophic factor; OD, optical density.

A

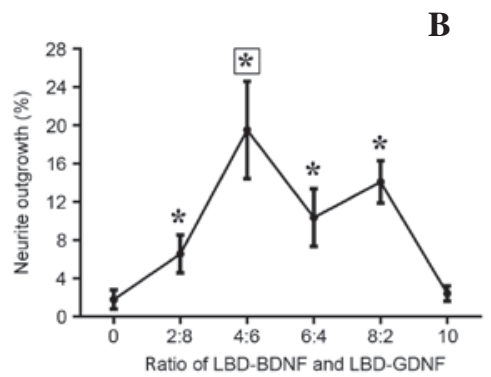

B

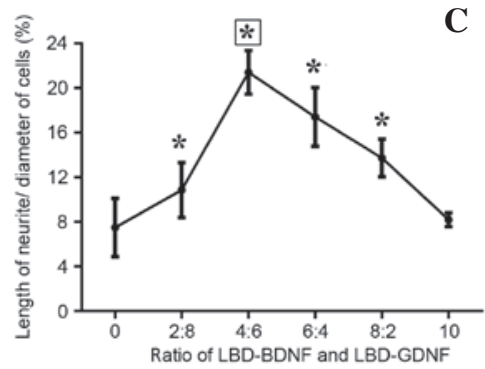

C

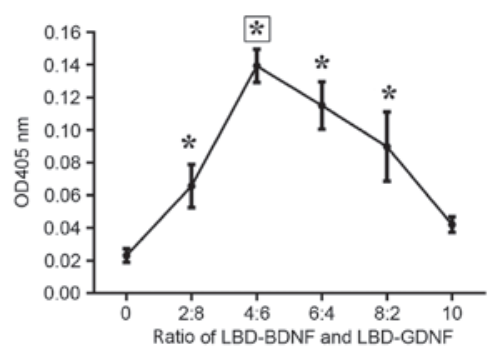

Figure 8. Bioactivity comparison of different porportions of recombinant proteins on laminin in vitro. Effect of different porportions of recombinant proteins on (A) neurite outgrowth and (B) the the ratio of neurite length/cell diameter in PC12 cells. (C) Effect of different proportions of recombinant proteins on cell survival in PC12 cells by Cell Counting kit-8 assay. Blank controls (ratio 0 or 10) indicates cells were cultured under similar conditions with the exception that LBD-BDNF or LBD-GDNF was omitted. Data are presented as mean \pm standard deviation $(n=6)$. "P $<0.05$ vs. NAT control. The asterisk within a square indicates the optimum ratio of mixing to promote growth. NAT, native; LBD, laminin-binding domain; BDNF, brain-derived neurotrophic factor; GDNF, glial cell line-derived neurotrophic factor; OD, optical density.

costs with these methods would all be increased. Moreover, the presence of redundant neurotrophic factors could cause adverse effects (7).

Laminin, a critical component of the ECM in the PNS, has been shown to affect neuronal behavior, including migration, neurite outgrowth, proliferation, and central synaptic differentiation $(20,36)$. A previous study has shown that LBD-BDNF can support marked neuroprotective function following middle cerebral artery occlusion (13). Therefore, in an attempt to retain neurotrophic factors at the site of injury and enhance nerve regeneration, laminin was chosen as a target for BDNF and GDNF in the present study. Whether 

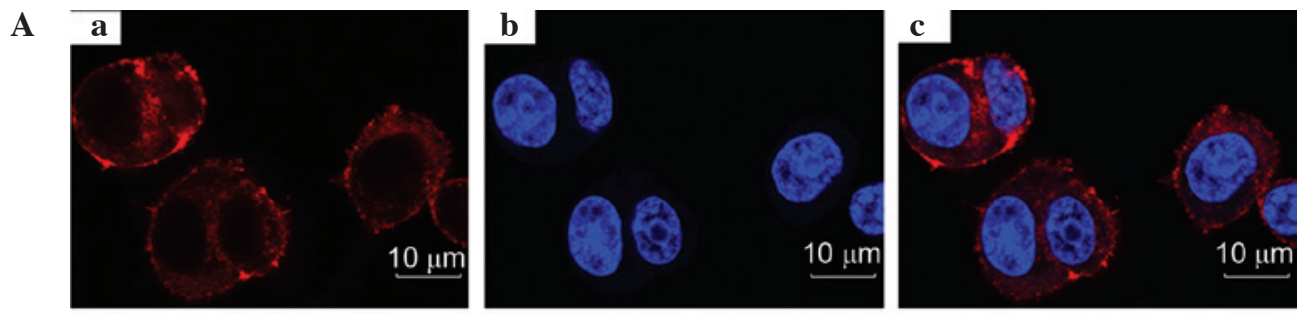

B
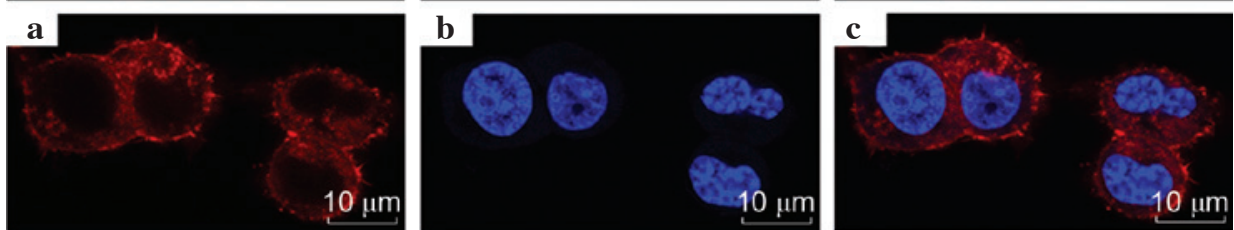

C
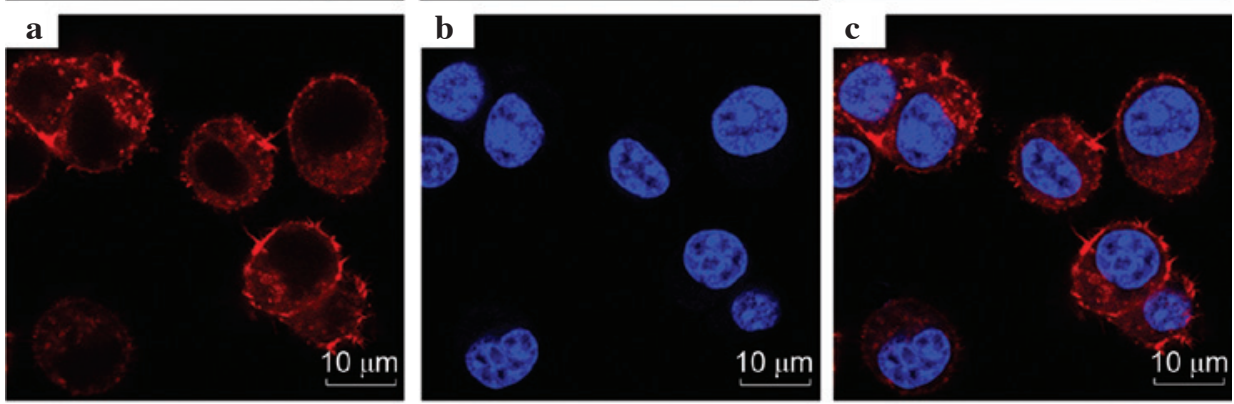

D
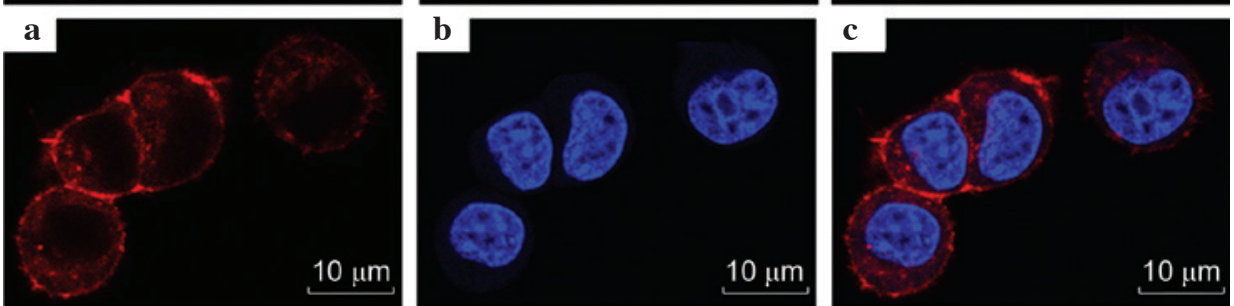

$\mathbf{E}$
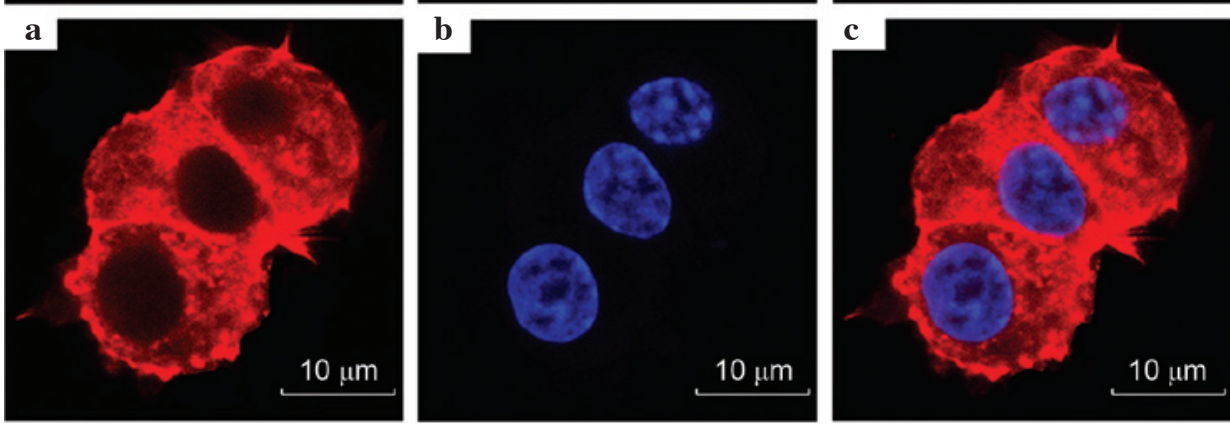

$\mathbf{F}$
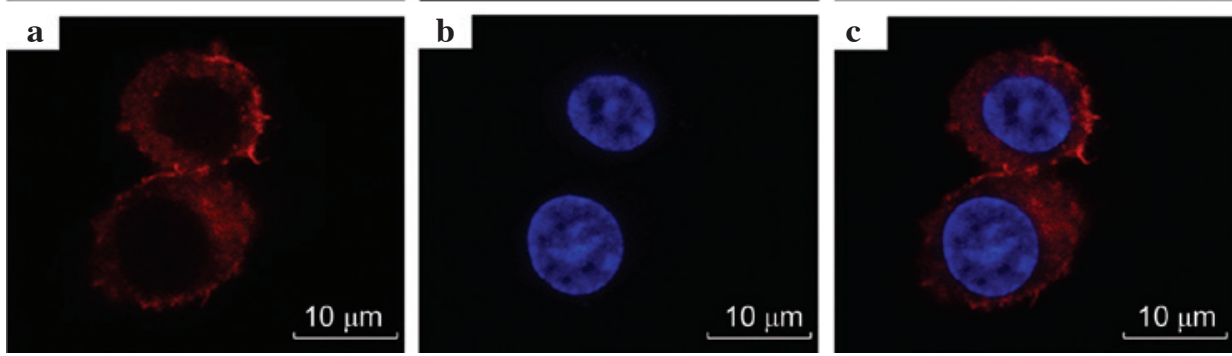

Figure 9. Images of PC12 cell (Aa) cytoskeletons; (Ab) nucleim, or (Ac) overlays, viewed by confocal fluorescent microscopy following treatment with different proportions of LBD-BDNF and LBD-GDNF. (A) Only LBD-BDNF, LBD-GDNF and LBD-BDNF in a (B) 1:4 ratio, (C) 2:3 ratio, (D) 3:2 ratio and (E) 4:1 ratio, and (F) only LBD-GDNF. NAT, native; LBD, laminin-binding domain; BDNF, brain-derived neurotrophic factor; GDNF, glial cell line-derived neurotrophic factor.

LBD-BDNF and LBD-GDNF had synergistic effects was also evaluated.

Agrin is a synapse organizer that promotes acetylcholine receptor clustering at the neuromuscular junction. Research has shown that NtA has high affinity with the coiled-coil domain of laminin (18). Thus, in order to target BDNF and GDNF to laminin, $\mathrm{NtA}$ was fused to the two neurotrophic factors to create LBD-BDNF and LBD-GDNF in the present study. As expected, compared with NAT-BDNF and NAT-GDNF, LBD-BDNF and LBD-GDNF presented comparable laminin-binding 
ability and demonstrated neuroprotective activities. In the laminin-binding assay of LBD-BDNF and LBD-GDNF, neurotrophic factors with NtA showed stronger binding abilities to laminin compared with those without NtA. At the same concentration, neurotrophic factors with NtA could be targeted to laminin, and effectively avoided being washed away or extensively diluted by extracellular fluids. Thus, LBD-BDNF or LBD-GDNF served as targeted proteins for maintaining effective concentrations of neurotrophic factors.

Next, PC12 cells were used for evaluating the bioactivities of LBD-BDNF and LBD-GDNF through neurite outgrowth and the CCK- 8 assay. The results showed that LBD-BDNF and LBD-GDNF maintained the bioactivities of BDNF and GDNF. Additionally, neurotrophic factors with NtA retained higher concentrations and bioactivities compared with those without NtA. It may be concluded that this was due to a difference in laminin-binding affinity between these proteins. Further, these findings showed that NtA enabled BDNF and GDNF target laminin.

Finally, LBD-BDNF and LBD-GDNF were mixed together in varying ratios to detect which ratio of mixing was the most conducive for the growth of PC12 cells. Optimal results were observed when LBD-BDNF and LBD-GDNF were mixed in a ratio of 4:6. Moreover, it was found that a mixture of LBD-BDNF and LBD-GDNF promoted the growth of PC12 cells to a greater extent than the use of either LBD-BDNF or LBD-GDNF alone at the corresponding concentration.

The present in vitro experiments clearly showed that these fusion proteins had certain advantages over native ones. Thus, the above findings provide evidence for the use of fusion proteins to immobilize a molecule, such as BDNF or GDNF, on a laminin-coated surface.

There are many methods for the application of exogenous neurotrophic factors; however, it appears that the most effective approaches for maintaining the survival of motor neurons and promoting their axonal regeneration is via routes that ensure sufficient concentrations and the continuous function of neurotrophic factors. The disadvantages of locally injecting neurotrophic factors include poor outcome, short-lived benefits, and side effects (30). If the administration of such factors could be controlled effectively and accurately at the injured area, they could be more safe and effective. In addition, there are few studies that have reported on the application of neurotrophic factors to regeneration of the RLN. Further basic research and advancements in the clinical development of RLN repair technology should allow for a better understanding of this approach. Moreover, clinical and basic research of the administration of neurotrophic factors via this technology is likely to promote the treatment of RLN injury and other diseases of the nervous system.

In summary, in the current study, the efficacy of a laminin-targeting peripheral nerve injury repair system was evaluated. It was found that this system maintained high concentrations and bioactivities of neurotrophic factors when compared with non-targeted controls. Moreover, when the ratio of mixing of LBD-BDNF and LBD-GDNF was 4:6, the ability of these factors to promote growth was superior to the combination of these factors in other ratios; this finding might be important when developing therapeutic strategies for repairing peripheral nerve injury.

\section{Acknowledgements}

This study was supported by a grant from the National Natural Science Foundation of China (no. 81271067).

\section{References}

1. Tessema B, Roark RM, Pitman MJ, Weissbrod P, Sharma S and Schaefer SD: Observations of recurrent laryngeal nerve injury and recovery using a rat model. Laryngoscope 119: 1644-1651, 2009.

2. Choi JS, Oh SH, An HY, Kim YM, Lee JH and Lim JY: Functional regeneration of recurrent laryngeal nerve injury during thyroid surgery using an asymmetrically porous nerve guide conduit in an animal model. Thyroid 24: 52-59, 2014.

3. Araki K, Shiotani A, Watabe K, Saito K, Moro K and Ogawa K: Adenoviral GDNF gene transfer enhances neurofunctional recovery after recurrent laryngeal nerve injury. Gene Ther 13: 296-303, 2006

4. Navarro X, Vivó M and Valero-Cabré A: Neural plasticity after peripheral nerve injury and regeneration. Prog Neurobiol 82: 163-201, 2007.

5. Lundborg G: A 25-year perspective of peripheral nerve surgery: Evolving neuroscientific concepts and clinical significance. J Hand Surg Am 25: 391-414, 2000.

6. Woodson GE: Spontaneous laryngeal reinnervation after recurrent laryngeal or vagus nerve injury. Ann Otol Rhinol Laryngol 116: 57-65, 2007.

7. Guan J, Zhang B, Zhang J, Ding W, Xiao Z, Zhu Z, Han Q, Wu C, Sun $\mathrm{Y}$, Tong $\mathrm{W}$, et al: Nerve regeneration and functional recovery by collagen-binding brain-derived neurotrophic factor in an intracerebral hemorrhage model. Tissue Eng Part A 21: 62-74, 2015.

8. Henderson CE, Phillips HS, Pollock RA, Davies AM, Lemeulle C, Armanini M, Simmons L, Moffet B, Vandlen RA, Simpson LC corrected to Simmons L, et al: GDNF: A potent survival factor for motoneurons present in peripheral nerve and muscle. Science 266: 1062-1064, 1994.

9. Li L, Wu W, Lin LF, Lei M, Oppenheim RW and Houenou LJ: Rescue of adult mouse motoneurons from injury-induced cell death by glial cell line-derived neurotrophic factor. Proc Natl Acad Sci USA 92: 9771-9775, 1995.

10. Yan Q, Matheson C and Lopez OT: In vivo neurotrophic effects of GDNF on neonatal and adult facial motor neurons. Nature 373: 341-344, 1995.

11. Sakamoto T, Watabe K, Ohashi T, Kawazoe Y, Oyanagi K, Inoue $\mathrm{K}$ and Eto $\mathrm{Y}$ : Adenoviral vector-mediated GDNF gene transfer prevents death of adult facial motoneurons. Neuroreport 11: 1857-1860, 2000.

12. Yanamoto H, Nagata I, Sakata M, Zhang Z, Tohnai N, Sakai H and Kikuchi H: Infarct tolerance induced by intra-cerebral infusion of recombinant brain-derived neurotrophic factor. Brain Res 859: 240-248, 2000.

13. Han Q, Li B, Feng H, Xiao Z, Chen B, Zhao Y, Huang J and Dai J: The promotion of cerebral ischemia recovery in rats by laminin-binding BDNF. Biomaterials 32: 5077-5085, 2011.

14. Longo FM, Hayman EG, Davis GE, Ruoslahti E, Engvall E, Manthorpe $\mathrm{M}$ and Varon S: Neurite-promoting factors and extracellular matrix components accumulating in vivo within nerve regeneration chambers. Brain Res 309: 105-117, 1984.

15. Lander AD, Fujii DK and Reichardt LF: Purification of a factor that promotes neurite outgrowth: Isolation of laminin and associated molecules. J Cell Biol 101: 898-913, 1985.

16. Martini R: Expression and functional roles of neural cell surface molecules and extracellular matrix components during development and regeneration of peripheral nerves. J Neurocytol 23: 1-28, 1994.

17. Fu SY and Gordon T: The cellular and molecular basis of peripheral nerve regeneration. Mol Neurobiol 14: 67-116, 1997.

18. Mascarenhas JB, Rüegg MA, Winzen U, Halfter W, Engel J and Stetefeld J: Mapping of the laminin-binding site of the N-terminal agrin domain (NtA). EMBO J 22: 529-536, 2003.

19. Gu Z, Weidenhaupt M, Ivanova N, Pavlov M, Xu B, Su ZG and Janson JC: Chromatographic methods for the isolation of, and refolding of proteins from, Escherichia coli inclusion bodies. Protein Expr Purif 25: 174-179, 2002.

20. Sun W, Sun C, Zhao H, Lin H, Han Q, Wang J, Ma H, Chen B, $\mathrm{Xiao} \mathrm{Z}$ and Dai J: Improvement of sciatic nerve regeneration using laminin-binding human NGF-beta. PLoS One 4: e6180, 2009. 
21. Yang XN, Jin YQ, Bi H, Wei W, Cheng J, Liu ZY, Shen Z, Qi ZL and Cao Y: Peripheral nerve repair with epimysium conduit. Biomaterials 34: 5606-5616, 2013.

22. Levi-Montalcini R: The nerve growth factor: Thirty-five years later. Biosci Rep 7: 681-699, 1987.

23. Sun W, Lin H, Chen B, Zhao W, Zhao Y, Xiao Z and Dai J: Collagen scaffolds loaded with collagen-binding NGF-beta accelerate ulcer healing. J Biomed Mater Res A 92: 887-895, 2010.

24. Sun W, Lin H, Chen B, Zhao W, Zhao Y and Dai J: Promotion of peripheral nerve growth by collagen scaffolds loaded with collagen-targeting human nerve growth factor-beta. J Biomed Mater Res A 83: 1054-1061, 2007.

25. Sun W, Sun C, Lin H, Zhao H, Wang J, Ma H, Chen B, Xiao Z and Dai J: The effect of collagen-binding NGF-beta on the promotion of sciatic nerve regeneration in a rat sciatic nerve crush injury model. Biomaterials 30: 4649-4656, 2009.

26. Kitajima T, Terai $\mathrm{H}$ and Ito $\mathrm{Y}$ : A fusion protein of hepatocyte growth factor for immobilization to collagen. Biomaterials 28 : 1989-1997, 2007

27. Han Q, Sun W, Lin H, Zhao W, Gao Y, Zhao Y, Chen B, Xiao Z, $\mathrm{Hu}$ W, Li Y, et al: Linear ordered collagen scaffolds loaded with collagen-binding brain-derived neurotrophic factor improve the recovery of spinal cord injury in rats. Tissue Eng Part A 15 : 2927-2935, 2009.

28. Han Q, Jin W, Xiao Z, Ni H, Wang J, Kong J, Wu J, Liang W, Chen L, Zhao Y, et al: The promotion of neural regeneration in an extreme rat spinal cord injury model using a collagen scaffold containing a collagen binding neuroprotective protein and an EGFR neutralizing antibody. Biomaterials 31: 9212-9220, 2010.

29. Liang W, Han Q, Jin W, Xiao Z, Huang J, Ni H, Chen B, Kong J, Wu J and Dai J: The promotion of neurological recovery in the rat spinal cord crushed injury model by collagen-binding BDNF. Biomaterials 31: 8634-8641, 2010.
30. Guan J, Tong W, Ding W, Du S, Xiao Z, Han Q, Zhu Z, Bao X, Shi $\mathrm{X}, \mathrm{Wu} \mathrm{C}$, et al: Neuronal regeneration and protection by collagen-binding BDNF in the rat middle cerebral artery occlusion model. Biomaterials 33: 1386-1395, 2012.

31. Han S, Wang B, Jin W, Xiao Z, Chen B, Xiao H, Ding W, Cao J, Ma F, Li X, et al: The collagen scaffold with collagen binding BDNF enhances functional recovery by facilitating peripheral nerve infiltrating and ingrowth in canine complete spinal cord transection. Spinal Cord 52: 867-873, 2014

32. Han S, Wang B, Jin W, Xiao Z, Li X, Ding W, Kapur M, Chen B, Yuan B, Zhu T, et al: The linear-ordered collagen scaffold-BDNF complex significantly promotes functional recovery after completely transected spinal cord injury in canine. Biomaterials 41: 89-96, 2015.

33. Cao J, Sun C, Zhao H, Xiao Z, Chen B, Gao J, Zheng T, Wu W, Wu S, Wang J and Dai J: The use of laminin modified linear ordered collagen scaffolds loaded with laminin-binding ciliary neurotrophic factor for sciatic nerve regeneration in rats. Biomaterials 32: 3939-3948, 2011

34. Moro K, Shiotani A, Watabe K, Takeda Y, Saito K, Mori Y and Ogawa K: Adenoviral gene transfer of BDNF and GDNF synergistically prevent motoneuron loss in the nucleus ambiguus. Brain Res 1076: 1-8, 2006.

35. Shiotani A, Saito K, Araki K, Moro K and Watabe K: Gene therapy for laryngeal paralysis. Ann Otol Rhinol Laryngol 116: 115-122, 2007.

36. Chen ZL and Strickland S: Laminin gamma 1 is critical for Schwann cell differentiation, axon myelination, and regeneration in the peripheral nerve. J Cell Biol 163: 889-899, 2003. 